04

\title{
Измерения колебаний дрейфового тока в двигателях с замкнутым дрейфом электронов
}

\author{
(C) T. Чернышёв,,$^{1,2}$ Д. Криворучко, ${ }^{2}$ А. Скрылёв ${ }^{2}$ \\ ${ }^{1}$ Объединенный институт высоких температур РАН, \\ 125412 Москва, Россия \\ ${ }^{2}$ Московский фризико-технический институт, \\ 141701 Долгопрудный, Московская обл., Россия \\ e-mail: thunarux@gmail.com
}

(Поступило в Редакцию 24 июля 2017 г.)

Индукционный метод для измерения дрейфового тока в двигателях с анодным слоем (ДАС) был использован для измерения интегрального искажения магнитного поля в канале стационарного плазменного двигателя (СПД) малой мощности. В работе подробно описана методология эксперимента, в результате которого определяется интегральный уровень колебаний магнитного поля в узком канале двигателя с замкнутым дрейфом электронов. Полученные данные показали, что в СПД зажигание разряда сопровождается полным размагничиванием разрядного промежутка дрейфовым током (отношение собственного магнитного поля к внешнему достигает 90\%), для ДАС наблюдается аналогичный эффект. При этом, в обоих двигателях полный ток разряда на полтора порядка превышает значения, характерные для стационарного режима, а свечение плазмы наблюдается не только внутри, но и за срезом разрядного канала. Проведены измерения колебаний дрейфового тока в установившихся режимах. В частности, показано, что при наличии сильных колебаний в области малых магнитных полей $\left(10^{-2} \mathrm{Gs}\right)$ искажение магнитного поля дрейфовым током может достигать $33 \%$. В режиме номинальной работы $\left(2 \cdot 10^{2} \mathrm{Gs}\right)$ оно не превышает $8 \%$. Характер колебаний и режимы работы в СПД и ДАС отличаются при выходе за границу оптимальной работы. Так, в СПД отсутствует критическое магнитное поле, при котором происходит переход в „турбулентный“ режим. В целом ток разряда слабее зависит от величины магнитного поля.

DOI: $10.21883 /$ JTF.2018.05.45899.2458

\section{1. Введение}

Двигатели с замкнутым дрейфом электронов (также известные как холловские электрореактивные двигатели, ХЭРД) - это азимутально-симметричные устройства, где разряд горит в кольцевом канале, образованном полюсами магнитопровода. Внешнее магнитное поле имеет преимущественно радиальную составляющую, а электрическое поле в основном параллельно оси двигателя. Электроны осциллируют в скрещенных полях между катодной и анодной областями разряда, формируя дрейфовый ток, замкнутый в азимутальном направлении („холловский ток“), и ионизируют поступающий из анода газ. Существуют два основных варианта двигателей с замкнутым дрейфом электронов: стационарный плазменный двигатель (СПД, двигатель Морозова) и двигатель с анодным слоем (ДАС, двигатель Жаринова). Для СПД характерен протяженный разрядный канал, выполненный из диэлектрика; для ДАС короткий разрядный канал ограничен проводящими стенками. В современных моделях СПД часто используется „гибридная“ схема с проводящей вставкой под плавающим потенциалом в прианодной области. Это позволяет снизить потери энергии на стенки вследствие ухода электронов.

Знание распределения магнитного поля необходимо для понимания физики процессов, протекающих в ХЭРД, а также для задач численного моделирования и ряда диагностических методов. Оно определяет размер циклоидных траекторий электронов и поперечную проводимость, от величины которых напрямую зависит эффективность работы двигателя. Магнитное поле в ХЭРД складывается из постоянной внешней компоненты и искажения, вносимого дрейфовым током заряженных частиц. Обычно, влиянием полей плазмы на внешнее поле пренебрегают, так как в большинстве случаев оно не значительно, в частности, авторы [1] проводили локальные измерения усредненного по времени магнитного поля в квазистационарном режиме работы СПД датчиком Холла и с помощью расчета по данным о плотности плазмы и распределении потенциала, полученных с зонда Ленгмюра. Измерения показали, что искажение магнитного поля мало (менее $5 \cdot 10^{-4} \mathrm{Gs}$ при постоянной составляющей $\left.1.2 \cdot 10^{-2} \mathrm{Gs}\right)$. В работе [2] использовался набор миниатюрных катушек для измерения распределения моментальной локальной плотности дрейфового тока в СПД при помощи быстрого $(<1 \mu \mathrm{s})$ прерывания разрядного тока, измерена локальная плотность дрейфового тока $\sim 30 \mathrm{~A} / \mathrm{cm}^{2}$, а также измерялась ВЧ составляющая, связанная с изменением подвижности электронов в плазме. В работе [3] исследовалось взаимодействие $\mathrm{c}$ дрейфового тока $\mathrm{c}$ цепями питания катушек намагничивания. В частности, рассматривался резонанс колебаний магнитного поля с собственной частотой магнитной системы. 
При использовании инвазивных методов габариты зонда должны быть много меньше разрядной камеры, поэтому их применение затруднено в устройствах малых размеров и при больших мощностях разряда, кроме того, зонд вносит возмущение и не позволяет проводить длительные измерения. Использование миниатюрных катушек достаточно трудоемко и затруднительно в случае двигателей с коротким каналом, например в двигателях с анодным слоем (ДАС). В работе [4] был предложен индукционный метод интегрального измерения дрейфового тока. Вместо установки дополнительных катушек для снятия сигнала использовалась катушка намагничивания, что не требовало каких-либо изменений в конструкции двигателя и позволяло снимать данные при малых габаритах канала. Измерения проводились с помощью аппаратного интегрирования переменной ЭДС, возникающей на катушке в момент быстрого прерывания разрядного тока. При этом предварительно проводилась калибровка измерительной системы с помощью пробной катушки, имитирующей дрейфовый ток. Полученные измерения позволили восстановить тягу разряда в момент прерывания тока. В [5] индукционный метод был усовершенствован для получения осциллограмм переменной составляющей дрейфового тока в ДАС. В частности, были проведены измерения колебаний дрейфового тока в различных режимах горения разряда и в момент зажигания. В настоящей работе индукционный метод используется для измерения переменной составляющей дрейфового тока в СПД малой тяги в момент зажигания и в режимах с сильными колебаниями. Полученные результаты сравниваются с данными по ДАС из работ $[5,6]$.

\section{2. Теория}

Можно оценить искажение магнитного поля, вносимое дрейфовым током с помощью закона Ампера-Максвелла, пренебрегая производными по времени и ограничиваясь рассмотрением вдоль оси разрядного канала:

$$
\delta B=\frac{4 \pi}{d c} \cdot J_{\theta}
$$

где $d$ - радиальная ширина разрядного канала, $c$ скорость света, $J_{\theta}$ - дрейфовый ток. Из-за наличия магнитопровода дрейфовый ток в основном оказывает размагничивающий эффект на катодную область разряда. В квазистационарных режимах также имеет место связь реактивной тяги ионного пучка и усредненным по колебаниям дрейфовым током:

$$
\left\langle J_{\theta}\right\rangle=\frac{F_{c}}{2 \pi R_{0} B_{0}},
$$

где $F-$ реактивная тяга, $R_{0}-$ средний радиус разрядного канала, $B_{0}$ - внешнее магнитное поле. Выражение (1) и (2) означает, что тяга ускоренных в самосогласованном электрическом поле электронов передается на магнитопровод благодаря искажению магнитного поля дрейфовым током. В эксплуатируемых режимах $|\delta B| / B_{0}$ (размагничивание) не превышает нескольких процентов. Из (2) и (1) следует, что тяга пропорциональна размагничиванию. Так как тяга пропорциональна ионному току, при больших токах возможны ситуации, когда искажение магнитного поля будет велико. Например, в момент зажигания разряда, когда ток на порядок превышает номинальный [7-9], или при достаточно сильных колебаниях. Кроме того, в ряде случаев разряды такого типа могут переходить в неустойчивый режим при небольших отклонениях магнитного поля $\left((1-2) \cdot 10^{-3} \mathrm{Gs}\right)$ от номинального.

\section{3. Эксперимент}

В настоящей работе рассматривается СПД с проводящей вставкой, геометрия разрядной камеры и форма магнитного поля для которого показана на рис. 1, $a$. Параметры СПД: $R_{0}=22.5 \mathrm{~mm}, d=8 \mathrm{~mm}$, полная глубина канала от среза магнитопровода до анода $20 \mathrm{~mm}$, размер диэлектрических вставок $1 \mathrm{~cm}$. Результаты сравниваются с ДАС (макет ХРДПУ, разрабатываемый в ФГУП ФЭИ), чья разрядная камера и форма магнитного поля показаны на рис. $1, b$. Параметры ДАС: $R_{0}=22.5 \mathrm{~mm}$, средняя ширина разрядного канала $d=5 \mathrm{~mm}$, глубина канала от среза магнитопровода до анода $7 \mathrm{~mm}$, детали проведения эксперимента с ДАС описаны в [5].

Исследования СПД проводились в лаборатории плазменных двигателей МФТИ. Экспериментальный стенд включает в себя горизонтально расположенную цилиндрическую вакуумную камеру диаметром $1.8 \mathrm{~m}$ и длиной $4.5 \mathrm{~m}$. Высоковакуумная система откачки обеспечивает остаточное предельное давление $2 \cdot 10^{-5} \mathrm{~Pa}$. Испытания проводились при расходе газа через анод $Q_{A}=1 \mathrm{mg} / \mathrm{s}$. Рабочее давление по ксенону на номинальном режиме работы двигателя составляло $8 \cdot 10^{-3} \mathrm{~Pa}$. Для замыкания тока использовался стандартный полый катод. Электрическая схема измерений приведена на рис. 2,a. Для стабилизации разрядного напряжения используется $L C$-фильтр $(1.5 \mathrm{mH}, 100 \mu \mathrm{F})$.
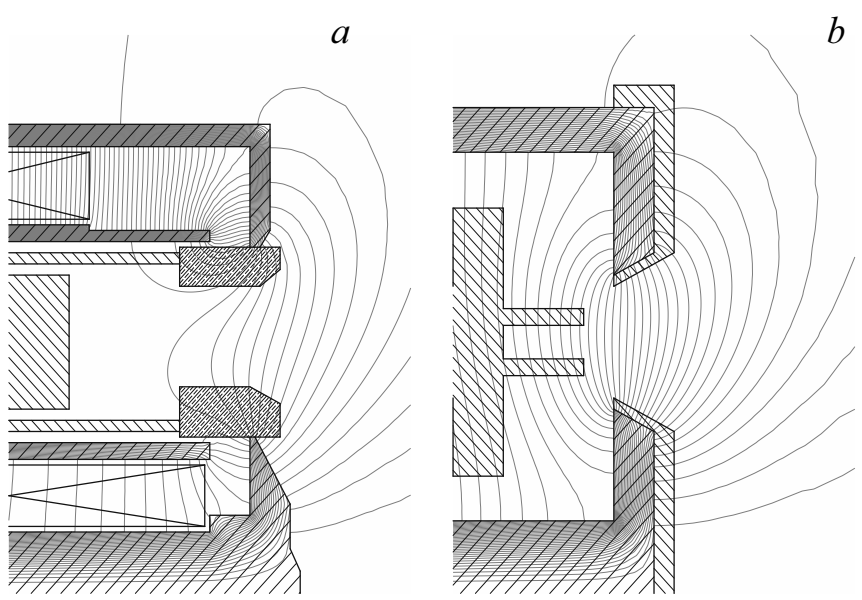

Рис. 1. Разряд камеры двигателей в масштабе (1:1). $a-$ СПД, $b-$ ДАС. 


\section{1. Методика измерений}

В зависимости от аксиального положения „центра масс“" дрейфового тока силовые линии его магнитного поля частично или полностью замыкаются через магнитопровод. Колебания дрейфового тока по амплитуде или его сдвиг в аксиальном направлении приводят к изменению магнитного потока через сердечник магнитопровода. Из закона электромагнитной индукции Фарадея следует, что это приводит к появлению переменной ЭДС в цепи катушки намагничивания:

$$
\delta U_{m} \propto d J_{\theta} / d t
$$

Тогда переменная составляющая магнитного поля может быть получена с помощью формулы:

$$
\delta J_{\theta}(t)=\mathscr{F}^{-1}\left(\frac{1}{K(\omega)} \mathscr{F}\left(\int_{0}^{t} \delta U_{m}(\tau) d \tau\right)\right)-a t-b,
$$

где $K(\omega)$ - коэффициент взаимной индукции, который зависит как от напряженности внешнего магнитного поля, так и от частоты $\omega$; $\mathscr{F}$ и $\mathscr{F}^{-1}-$ прямое и обратное преобразования Фурье; $a, b$ - корректирующие коэффициенты, устраняющие накапливающуюся при интегрировании систематическую ошибку, связанную с неточностью определения нуля сигнала. Для стабилизации тока катушек и исключения резонансного взаимодействия с дрейфовым током [3] в цепи катушек намагничивания вводились индуктивности $30 \mathrm{mH}$. Для выделения переменной составляющей измерение ЭДС на катушке проводилось через конденсатор $1000 \mu \mathrm{F}$ осциллографом с импедансом канала $50 \Omega$. Показания снимались с центральной катушки, соотношение токов между внутренней и внешней катушками всегда составляло $1: 0.88$. Коэффициенты взаимной индукции измерялись в ходе калибровочного эксперимента, когда дрейфовый ток имитировался с помощью модельной катушки с синусоидальным током, вводимой в разрядную камеру (рис. 2,b). Калибровочный эксперимент показал, что в исследуемой области частот $(1-100 \mathrm{kHz})$ коэффициент взаимной индукции линейно зависит от частоты и может быть описан формулой $1 / K(\omega)=\left(571 \omega[\mathrm{Hz}]+3 \cdot 10^{6}\right)$.

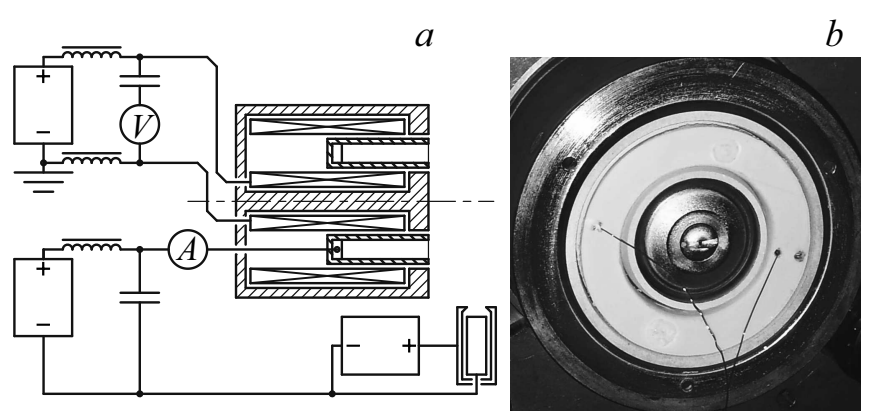

Рис. 2. $a-$ схема цепей, $b-$ измерение $K(\omega)$.
Вышеизложенный метод не позволяет различить изменение магнитного потока вследствие изменения дрейфового тока по амплитуде и вследствие его продольного движения. Из за неопределенности пространственного распределения дрейфового тока точность измерения колебаний магнитного поля составляет около $25 \%$. Оценка искажения магнитного поля проводится по формуле (1).

\section{2. Зажигание разряда}

На рис. 3, $а$ приведена осциллограмма зажигания разряда в СПД. Верхнее изображение - осциллограмма разрядного тока, нижнее - сигнал дрейфового тока, восстановленного по формуле (3). Для сравнения на рис. $3, b$ приведена аналогичная осциллограмма зажигания разряда в ДАС из работы [6]. Отметим, что в СПД анодный ток появляется с небольшой задержкой, которая связана, по-видимому, с зарядкой диэлектрических стенок канала и металлических вставок, а также конечным временем прохождения электронной лавины от катода к аноду через протяженный разрядный промежуток. Используя формулу (1), можно оценить искажение магнитного поля. Амплитуда „выброса“ дрейфового тока в СПД $\delta J_{\theta} \sim 120 \mathrm{~A}$, тогда $|\delta B| / B_{0} \sim 0.95$, т. е. происходит полное размагничивание разрядного промежутка. Для ДАС $\delta J_{\theta} \sim 80 \mathrm{~A}$, тогда $|\delta B| / B_{0} \sim 0.68$. Меньший ток и большая длительность процесса при зажигании ДАС связаны с использованием термоэмиссионных катодов (подробнее см. [9]).

Переходный процесс в момент зажигания разряда может быть интерпретирован следующим образом. В начальный момент времени разрядный канал равномерно заполнен газом, после зажигания заметная концентрация газа остается только вблизи анода. Можно по порядку величины оценить полное число заряженных частиц, участвующих в импульсе тока (с учетом ионизации натекающего газа):

$$
N_{A}=\int_{0}^{t_{1}} I_{A}(t) / e d t
$$

где $t_{1}$ - длительность зажигания. Сделав поправку на натекание газа, число частиц равно $N_{A 0}=$ $=N_{A}-t_{1} Q_{A}\left[\mathrm{~A}^{\mathrm{eq}}\right] / e, \quad\left(1 \mathrm{~A}^{\mathrm{eq}} \equiv 1.36 \mathrm{mg} / \mathrm{s}\right) . \quad$ Эксперимент показывает, что величина $N_{A 0}$ пропорциональна расходу. Для исследуемых макетов она составляет $N_{A 0} \sim Q_{A}\left[\mathrm{~A}^{\mathrm{eq}}\right] \cdot 10^{15}$ частиц. Отсюда можно сделать оценку аксиального размера области выгорания (предполагая, что $N_{A 0}$ составляют однозарядные ионы и электроны):

$$
L \sim u_{a} \frac{e}{2 Q_{A}\left[\mathrm{~A}^{\mathrm{eq}}\right]} \cdot N_{A 0},
$$

где $u_{a}$ - тепловая скорость атомов. Что для исследуемых макетов дает около $\sim 3 \mathrm{~cm}$ и сопоставимо со средним радиусом разрядного канала. Это означает, что ионизация происходит не только внутри разрядного 

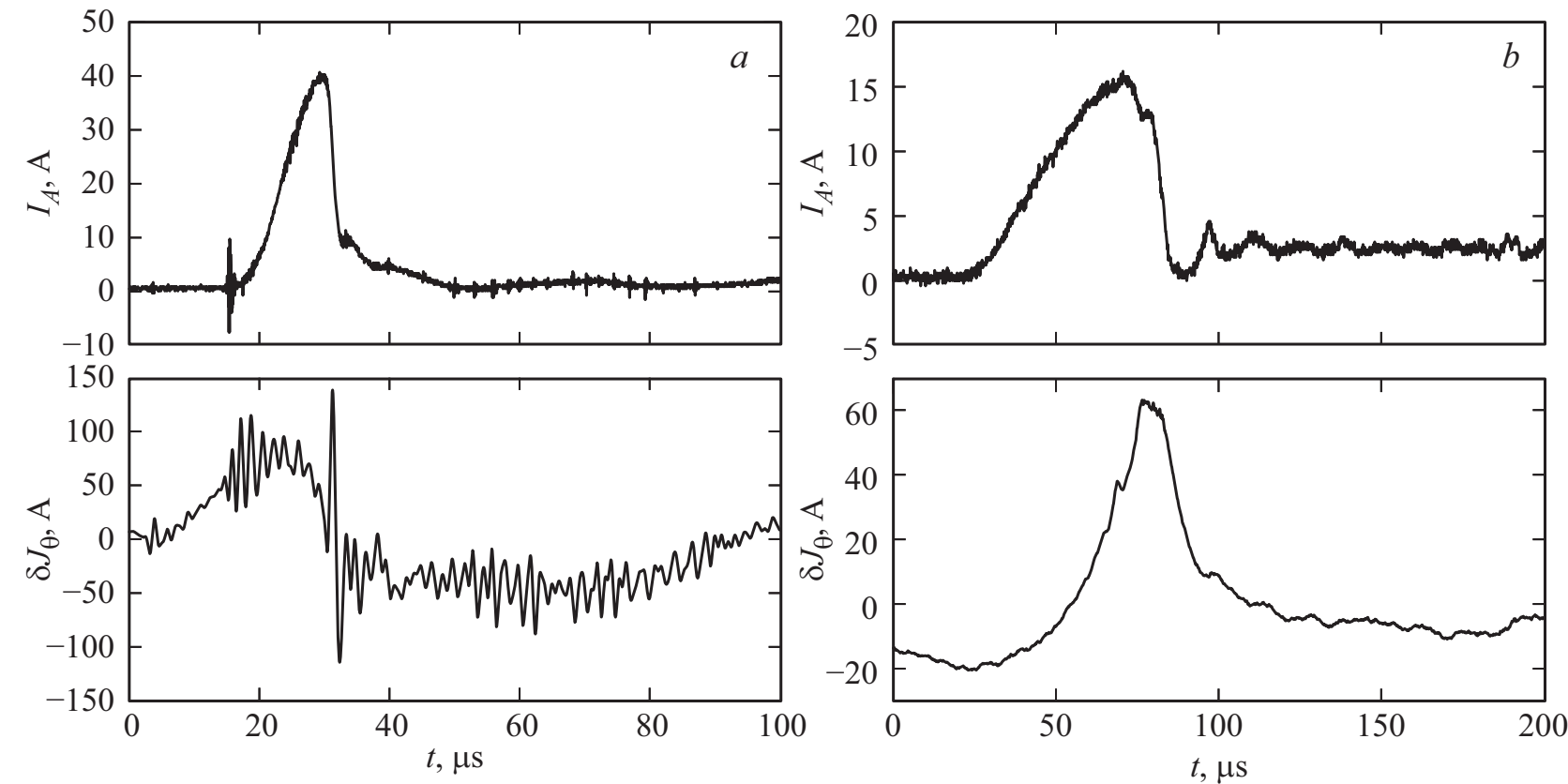

Рис. 3. Осциллограммы зажигания разряда. $a-$ СПД, $2 \cdot 10^{-2} \mathrm{Gs}, b-$ ДАС по данным $[6], B_{0}=2.9 \cdot 10^{-2} \mathrm{Gs}$.

канала, но и в достаточно большой области за срезом двигателя. Таким образом, „всплеск“ тока в момент зажигания обусловлен ионизацией „избыточных“ атомов. Можно оценить минимально необходимую емкость в анодной цепи, обеспечивающую протекание импульса тока: $C_{A}^{\min } \sim\left(e N_{A}-t_{1} I^{\max }\right) / \delta \phi_{A}$, где $\delta \phi \approx 50 \mathrm{~V}-$ максимальное падение напряжения, при котором не происходит погасания разряда; $I^{\max }-$ максимальный ток источника питания. При использовании ХЭРД на борту КА мощность источника питания близка мощности двигателя, поэтому $I^{\max }$ не более чем на $10 \%$ превышает ток номинального режима. Отсюда $C_{A}^{\min } \sim 2\left\langle I_{A}\right\rangle \cdot 10^{-6} \mathrm{~F}$, где $\left\langle I_{A}\right\rangle$ - номинальный ток двигателя.

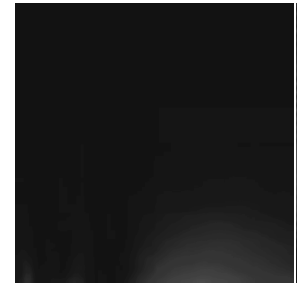

$t=0 \mu \mathrm{s}$

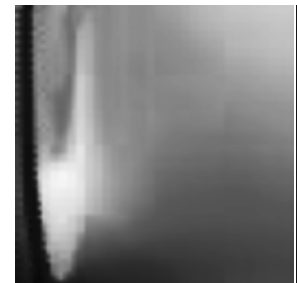

$t=13.63 \mu \mathrm{s}$

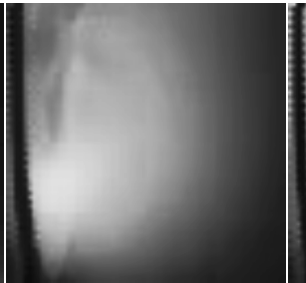

$t=4.54 \mu \mathrm{s}$

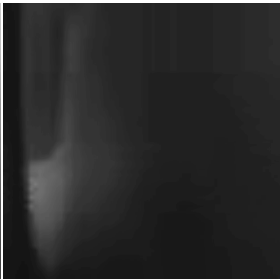

$t=18.18 \mu \mathrm{s}$

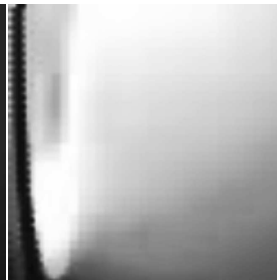

$t=9.09 \mu \mathrm{s}$

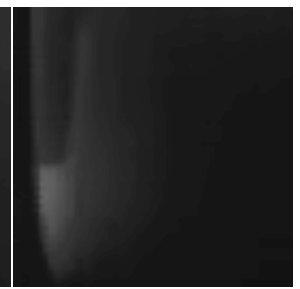

$t=27.27 \mu \mathrm{s}$
Рис. 4. Зажигание разряда.

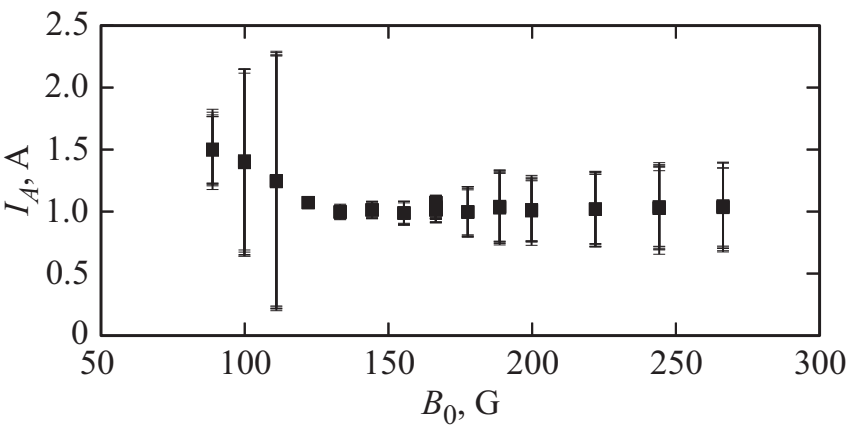

Рис. 5. Магнит-амперная характеристика разряда в СПД при $Q_{A}=1 \mathrm{mg} / \mathrm{s}, \phi_{A}=300 \mathrm{~V}$.

С целью визуализации процесса зажигания СПД проводилась высокоскоростная съемка разряда (рис. 4). В момент времени $t=0$ появляется облако плазмы, инжектированной из катода (слабое свечение справа снизу). После этого свечение возникает во всей разрядной области, достигая через $10 \mu \mathrm{s}$ максимальной яркости. В это время яркое свечение наблюдается как внутри канала, так и за его срезом. Примерно через $15-20 \mu \mathrm{s}$ интенсивность значительно падает и видимым остается свечение только внутри разрядного канала. С учетом того, что интенсивность излучения пропорциональна концентрации возбужденных частиц и соответственно возрастает с увеличением концентрации плазмы, изменение яркости во времени характеризует те же эффекты („всплеск“ тока, ионизация газа за срезом канала). 

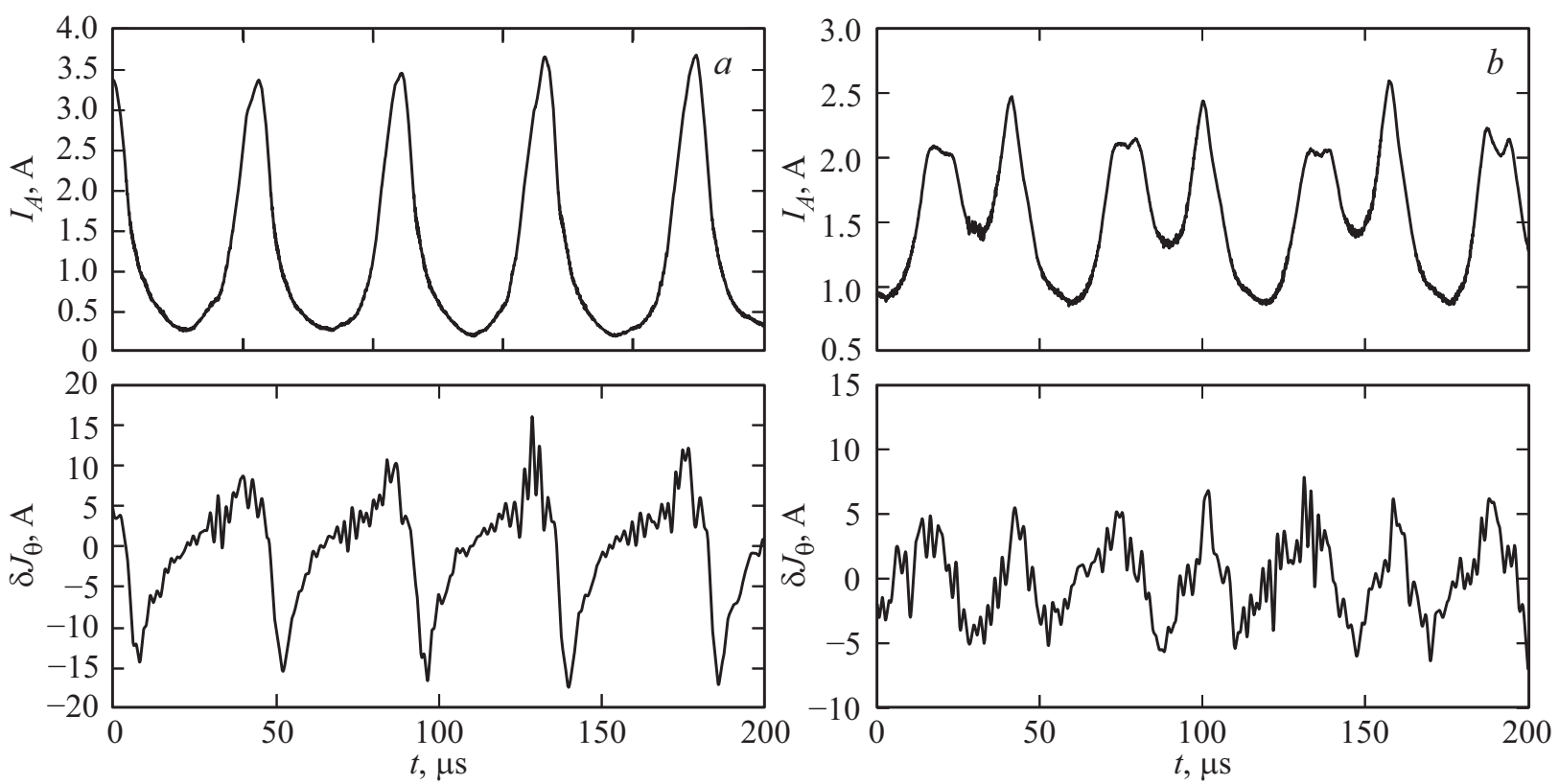

Рис. 6. Осциллограммы колебаний разряда в СПД, $Q_{A}=1 \mathrm{mg} / \mathrm{s}, \phi_{A}=300 \mathrm{~V} . a-B_{0}=10^{-2} \mathrm{Gs}, b-B_{0}=2 \cdot 10^{2} \mathrm{Gs}$.
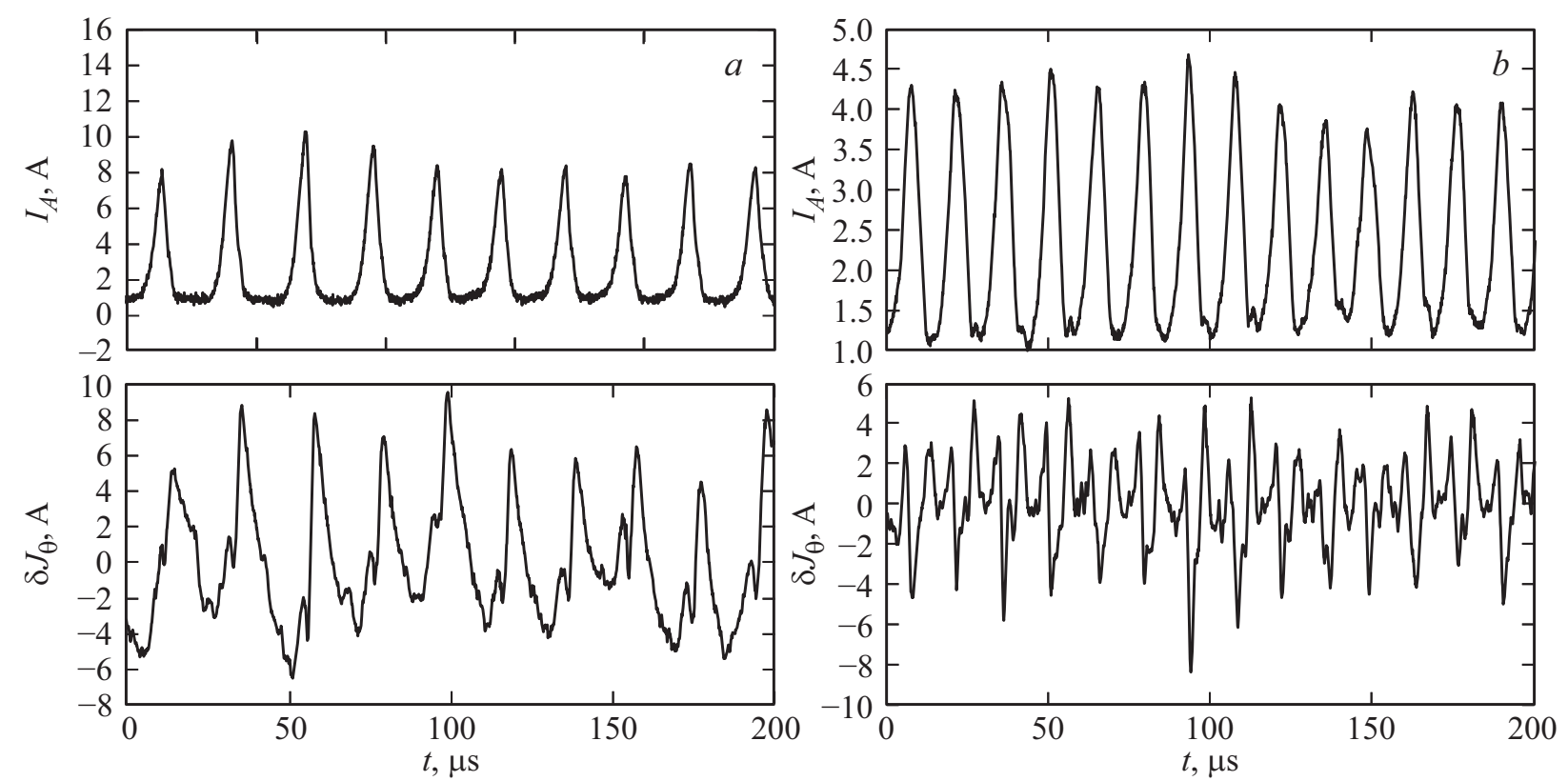

Рис. 7. Осциллограммы колебаний разряда в ДАС, $Q_{A}=1.8 \mathrm{mg} / \mathrm{s}, \phi_{A}=250 \mathrm{~V} . a-B_{0}=2.25 \cdot 10^{2} \mathrm{Gs}, b-B_{0}=2.7 \cdot 10^{-2} \mathrm{Gs}$.

\section{3. Установившийся режим}

Также рассматривались горение разряда в установившемся режиме при фиксированном напряжении $(300 \mathrm{~V})$ и варьировании магнитного поля. Измерялся анодный ток, переменная компонента дрейфового тока и тяга. На pис. 5 приведена зависимость анодного тока от магнитного поля (магнит-амперная характеристика разряда), разбросом показаны амплитуды колебаний. В области малых магнитных полей развиваются сильные колебания анодного тока - так называемые ,дыхательные моды“ (breathing modes) (рис. 6, a), в области высоких полей „ионизационные“ колебания (рис. $6, b)$. При этом тяга слабо зависит от величины магнитного поля. Так, при $B_{0}=10^{-2} \mathrm{Gs}$ и $300 \mathrm{~V}$ тяга составляет $1.62 \mathrm{~g}$, и при увеличении магнитного поля до $2 \cdot 10^{-2}$ Gs тяга монотонно возрастает до $1.78 \mathrm{~g}$, при дальнейшем увеличении магнитного поля тяга не меняется. Данная зависимость сохраняется при других напряжениях. Используя формулы (1) и (2) и считая, что $J_{\theta}=\left\langle J_{\theta}\right\rangle+\delta J_{\theta}$, можно оценить полное искажение магнитного поля. Так, в режиме „ионизационных“ колебаний $|\delta B| / B_{0} \approx 0.0092-0.087$, а 
в режиме „дыхательных мод“ $|\delta B| / B_{0} \approx 0.019-0.333$. Осциллограммы аналогичных измерений для ДАС показаны на рис. 7 (частота НЧ колебаний связана со временем пролета нейтрального атома через разрядный промежуток, поэтому для ДАС она оказалась выше). Для ДАС искажение магнитного поля в режиме ,дыхательных мод“ - $|\delta B| / B_{0} \approx 0.045-0.182$, в режиме „ионизационных“ колебаний $|\delta B| / B_{0} \approx 0.037-0.111$. Стоит отметить, что в отличие от СПД „дыхательные моды“ в рассматриваемой модели ДАС появляются только после зажигания разряда и в течение $\sim 1000 \mu$ s релаксируют к стационарному состоянию.

Поведение ДАС в области высоких магнитных полей значительно отличается от СПД - при превышении некоторого критического значения магнитного поля в ДАС возникает неустойчивость, сопровождающаяся появлением хаотических всплесков тока, - возникает „турбулентный режим“ $[5,10]$, который связывают со сменой механизма проводимости $[11,12]$. Подобный эффект полностью отсутствует в СПД - во всем диапазоне магнитных полей выше оптимального наблюдаются только „ионизационные“ колебания.

\section{Выводы}

Индукционный метод был успешно использован для измерения колебаний дрейфового тока в СПД малой тяги в момент зажигания и в режимах с сильными колебаниями. Приведено сравнение полученных результатов с аналогичными экспериментами, поставленными на ДАС. Измерения показали, что при зажигании разряда в СПД, так же как в ДАС, дрейфовый ток оказывается столь велик, что происходит значительное (90\%) размагничивание разрядного промежутка. При этом полный ток разряда на порядок превышает значения, характерные для стационарного режима, а интенсивная ионизация происходит не только внутри канала, но и за срезом двигателя. Однако, зажигание разряда в СПД отличается от ДАС - присутствует задержка между появлением сигнала дрейфового тока. Различная длительность зажигания связана с использованием разных типов катодов. Для СПД была проведена высокоскоростная съемка зажигания разряда, на которой наблюдались эффекты, аналогичные полученным в результате обработки осциллограмм: „всплеск“ тока и ионизация газа за срезом канала. Исследование установившихся режимов в СПД показало, что в области малых магнитных полей, где наблюдаются колебания тока большой амплитуды, максимальное искажение магнитного поля может достигать $33 \%$ от внешнего. В области номинальной работы искажение магнитного поля не превышает $8 \%$. Также в отличие от ДАС в СПД отсутствует верхняя граница магнитного поля, при котором разряд переходит в ,турбулентный“ режим.
Работа выполнена при финансовой поддержке гранта РНФ 14-50-00124"/"This work was supported by the Russian Science Foundation project 14-50-00124.

\section{Список литературы}

[1] Haas J.M., Gallimore A.D. Considerations on the Role of the Hall Current in a Laboratory-Model Thruster. In 37th Joint Propulsion Conference \& Exhibit, 8-11 July 2001.

[2] Cliff A. Thomas, Nicolas Gascon, Mark A. Cappelli. A Study of the Azimuthal Electron Drift in an $E \times B$ Discharge Using a Non-invasive Antenna Array. In 39th AIAA/ASME/SAE/ASEE Joint Propulsion Conf. and Exhibit, 20-23 July 2003.

[3] Da-ren Yu, Li-qiu Wei, Yong-jie Ding, Ke Han, Guo-jun Yan, Feng-yan Qi. // Plasm. Sourc. Science Technol. 2007. Vol. 16. N 4. P. 757.

[4] Новичков Д.Н., Ермилов А.Н., Коваленко А.Ю., Сапронова T.M. // TBT. 2005. Vol. 43. N 5. P. 691-696.

[5] Ермилов А.Н., Ерошенков В.Ф., Новичков Д.Н., Коваленко Ю.А., Сапронова Т.М., Чернышёв Т.В., Шумилин А.П. // TBT 2014. Vol. 52. N 3. P. 371-380.

[6] Чернышёв Т.В. Экспериментальные и численные исследования нарушения стационарности горения интенсивных разрядов с замкнутым дрейфом электронов. Автореф. канд. дис. ОИВТ РАН, 2016.

[7] Vial V., Mazouffre S., Prioul M., Pagnon D., Bouchoule A. // IEEE Transactions on Plasma Sci. 2005. Vol. 33. N 2.

[8] Ellison C.L., Raitses Y., Fisch N.J. // IEEE Trans. Plasma Sci. 2011. Vol. 39. N 11.

[9] Ермилов А.Н., Ерошенков В.Ф., Коваленко Ю.А., Королёв С.В., Чернышёв Т.В., Шумилин А.П. // ТВТ. 2013. Vol. 51. N 4. C. 497.

[10] Ермилов А.Н., Ерошенков В.Ф., Новичков Д.Н., Коваленко Ю.А., Сапронова Т.М., Королёв С.В., Чернышёв Т.В., Шумилин А.П. // ТВТ. 2013. Vol. 51. N 5. P. 670.

[11] Yamamoto Naoji, Komurasaki Kimiya, Arakawa Yoshihiro. Condition of Stable Operation in a Hall Thruster. In 28th International Electric Propulsion Conference, Toulouse, France, 2003.

[12] Takahashi Nanako, Yamamoto Naoji, Nakashima Hideki, Yokota Shigeru, Komurasaki Kimiya, Arakawa Yoshihiro. Investigation of Internal Plasma Structure in an Anode-layer Hall Thruster. In 30th Int. Electric Propulsion Conf. Florence, Italy, September 17-20 2007. 